\title{
ICTIOFAUNA DE POÇAS DE MARÉ EM TERRAÇOS CONSOLIDADOS DO LITORAL AMAZÔNICO BRASILEIRO
}

\author{
Rayane Gonçalves Aguiar ${ }^{1,2}$, Erick Cristofore Guimarães ${ }^{3,4,5}$, Pâmella Silva de Brito ${ }^{4,5}$, Felipe \\ Polivanov Ottoni ${ }^{1,2,4 *}$ \& Jorge Luiz Silva Nunes ${ }^{1,4,6}$
}

${ }^{1}$ Universidade Federal do Maranhão, Programa de Pós-Graduação em Biodiversidade e Conservação. Av. dos Portugueses 1966, Cidade Universitária do Bacanga, CEP 65080-805, São Luís, MA, Brasil.

${ }^{2}$ Universidade Federal do Maranhão, Centro de Ciências Agrárias e Ambientais, Laboratório de Sistemática e Ecologia de Organismos Aquáticos, BR-222, KM 04, Boa Vista, CEP 65500-000, Chapadinha, MA, Brasil.

${ }^{3}$ Universidade Estadual do Maranhão, Centro de Ciências Agrárias, Laboratório de Ictiofauna e Piscicultura Integrada, Av. Lourenço Vieira da Silva 1000, Cidade Universitária Paulo VI, Jardim São Cristovão, CEP 65055-310, São Luís, MA, Brasil.

${ }^{4}$ Universidade Federal do Maranhão, Programa de Pós-Graduação pela Rede de Biodiversidade e Biotecnologia da Amazônia Legal, Av. dos Portugueses 1966, Cidade Universitária do Bacanga, CEP 65080-805, São Luís, MA, Brasil.

${ }^{5}$ Universidade Federal do Oeste do Pará, Campus Tapajós, Instituto de Ciências da Educação, Programa de Pósgraduação Sociedade Natureza e Desenvolvimento, Rua Vera Paz, s/n, Salé, CEP 68040-470, Santarém, PA, Brasil.

${ }^{6}$ Universidade Federal do Maranhão, Departamento de Oceanografia e Limnologia, Laboratório de Organismos Aquáticos, Av. dos Portugueses 1966, Cidade Universitária do Bacanga, CEP 65080-805, São Luís, MA, Brasil.

E-mails: rayaneaguiar1706@gmail.com; erick.ictio@yahoo.com.br; pamellabrito@hotmail.com; fpottoni@gmail. com (*autor correspondente); jorge.nunes@ufma.br.

Resumo: As poças de maré são formadas pelo represamento de massas d'água durante a vazante e a sua troca ocorre ao longo de cada ciclo de marés, configurando em um ambiente explorado por várias espécies. A ictiofauna é um componente das poças de maré, algumas espécies são totalmente adaptadas (e.g. plasticidade fisológica, tamanho reduzido) e seu ciclo de vida ocorre apenas nas poças de maré. Contudo, muitas espécies utilizam as poças de maré em algumas fases do ciclo de vida para fins de alimentação, refúgio e reprodução. Considerando que os inventários são fundamentais para o conhecimento da biodiversidade, nosso estudo investigou a assembleia de peixes em poças de marés da Praia do Araçagy no período de outubro de 2016 a abril de 2017. No total, nós registramos 409 exemplares, pertencendo a 13 espécies, distribuídas em 11 famílias e sete ordens. Espécies residentes e estuarinas de importância comercial foram dominantes.

Palavras-chaves: Biodiversidade, região costeira, zona entremarés.

INTERTIDAL FISHES OF THE CEMENTED TERRACES FROM BRAZILIAN AMAZON COAST: Tidal pools are formed by the damming of marine water during an ebb tide and their exchange occurs along each tidal cycle, configuring an environment explored by several species. The ichthyofauna is one of the components of the tide pools, with some species being fully adapted (e. g. physiological plasticity, reduced size) and their life cycles occuring uniquely on this environment. However, most species use tide pools at some stage of their life cycle for feeding, refuge and reproduction. Considering the main role of faunal inventories for the increase in biodiversity knowledge, this study investigates the fish assemblage in tide 
pools at Praia do Araçagy from October 2016 to April 2017. In total, were recorded 409 specimens belonging to 13 species, distributed in 11 families and seven orders. Resident and estuarine species with commercial importance were dominant.

Keywords: Biodiversity, coastal region, intertidal zone.

As poças de maré são constituídas por corpos de água represados na areia, margens rochosas ou de arenito, e servem como ambiente para várias espécies de organismos (Nunes et al. 2011). Nas últimas duas décadas houve um aumento expressivo nas pesquisas sobre a ictiofauna que ocupam as poças de marés no Brasil, com enfoque na compreensão da estrutura de comunidades e características abióticas das poças (e.g. Barreiros et al. 2004, Cunha et al. 2007, 2008, Macieira \& Joyeux 2011, Macieira et al. 2011, Freitas \& Lotufo 2015, Machado et al. 2015, Pastro et al. 2016, Bezerra etal.2017). Pesquisas como inventários de espécies, processos ecológicos e monitoramento são essenciais para a manuntenção da saúde do meio ambiente (Machado et al. 2015) e relevantes para o conhecimento e conservação de espécies que ocorrem nas zonas entremarés.

O presente estudo teve o objetivo de fornecer uma lista de espécies que habitam as poças de maré formadas em terraços consolidados, em uma praia urbana com influência de macromarés típica do Litoral Amazônico Brasileiro. A praia do Araçagy (2²7'53,65”'S; 44¹1'50,99”'W) está localizada ao norte da Ilha do Maranhão, estado do Maranhão (Figura 1), sendo influenciada pelas macromarés semidiurnas do oceano Atlântico e características estuarinas do seu entorno, como o Golfão Maranhense.

Os exemplares foram coletados em poças de maré ao longo de toda extensão da zona entremarés da Praia do Araçagy (Figura 2) entre outubro de 2016 e abril de 2017, sob autorização do órgão fiscalizador (SISBIO 54949-1). Para a coleta da ictiofauna foi utilizada uma solução anestésica de mentol nas poças de maré (40 g/l) conforme metodologia proposta por Nunes et al. (2011), e em seguida os exemplares foram capturados com redes manuais. Posteriormente, os exemplares foram fixados em solução de formol a $10 \%$ por
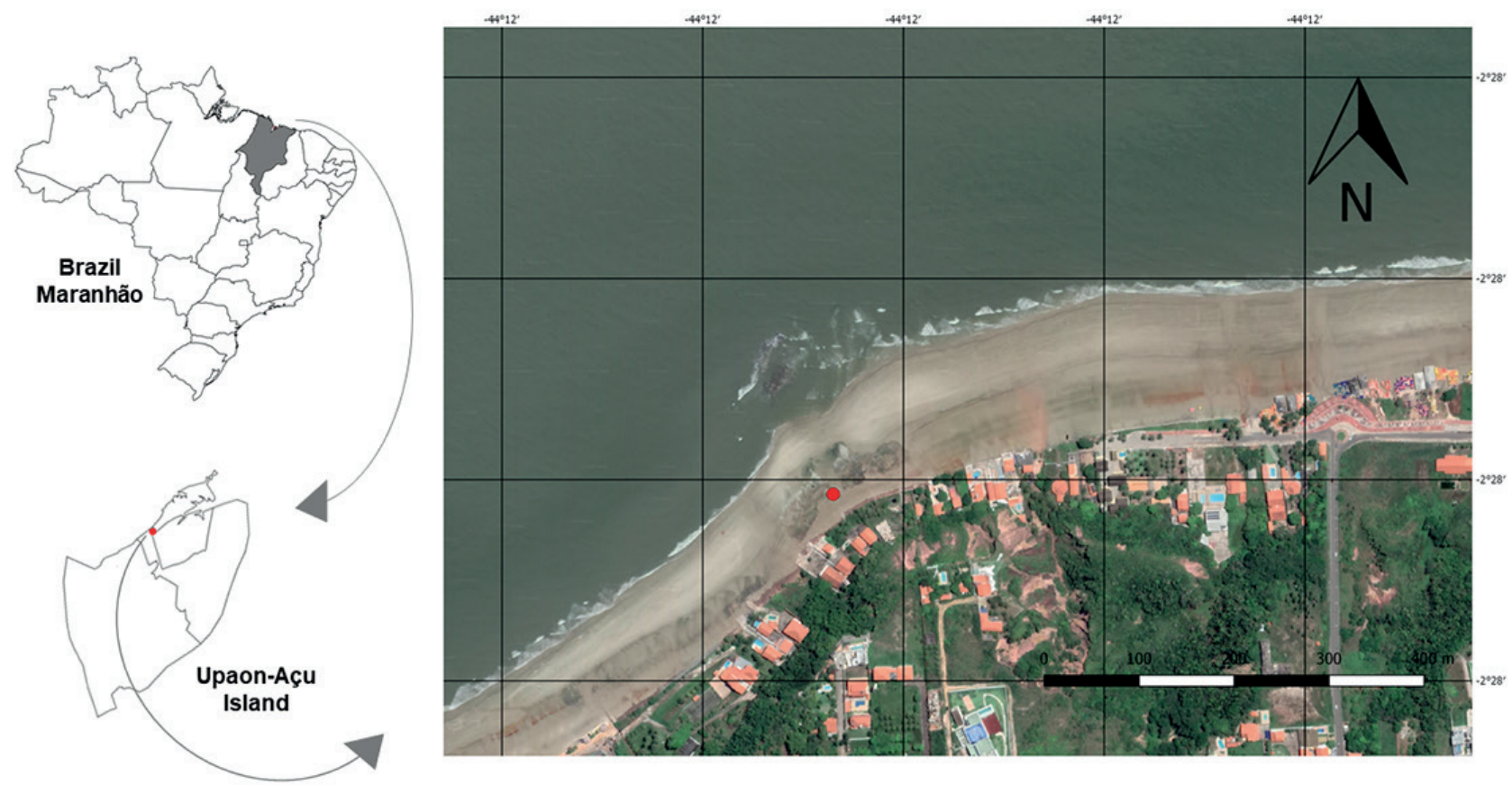

Figura 1. Imagem da área de estudo (ponto vermelho) na Praia do Araçagy, norte da ilha do Maranhão, estado do Maranhão, Brasil.

Figure 1. Image of the study area (red dot) in the Araçagy beach, north of the Maranhão island, state of Maranhão, Brazil. 

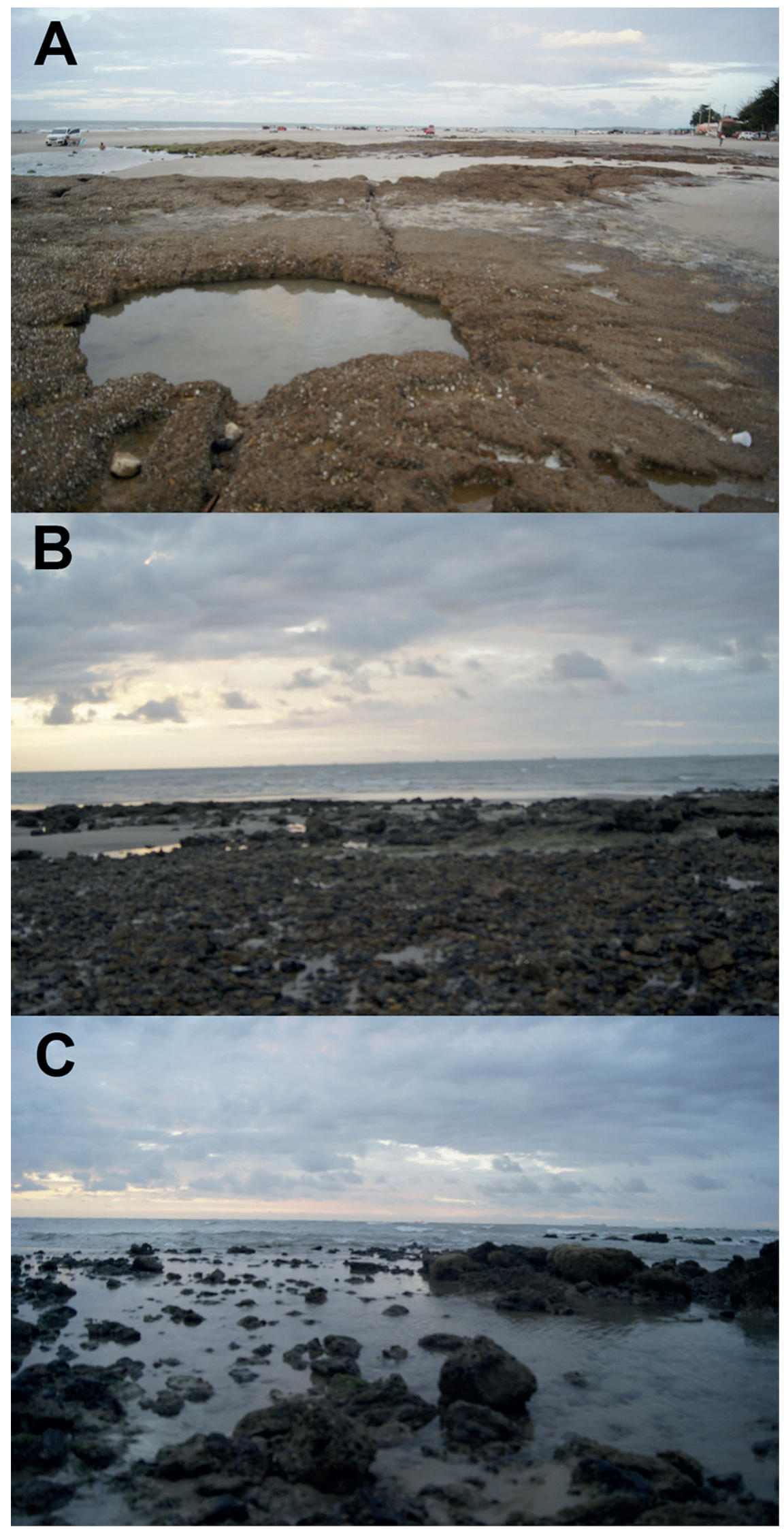

Figura 2. Poças de maré da Praia do Araçagy: A - supra-litoral, B- mesolitoral, e C- infra-litoral.

Figura 2. Tidal pools of Araçagy beach: A-supra-coastline, B- mesocoastline, and C - infra-coastline. 
20 dias e transferidos para uma solução de álcool a 70 \% para preservação.

O comprimento padrão (CP) de cada espécime foi medido com um paquímetro digital com precisão de $0,1 \mathrm{~mm}$, enquanto o peso total individual (PT) foi registrado utilizando uma balança digital com precisão de 0,01 g. As espécies foram identificadas ao menor nível taxonômico possível, consultando Carpenter (2002a, b). A classificação taxonômica foi baseada nas compilações propostas por Fricke et al. (2021). O material coletado foi depositado na Coleção Ictiológica do Centro de Ciências Agrárias e Ambientais da Universidade Federal do Maranhão (CICCAA).

Foram registrados 409 exemplares, pertencentes à 13 espécies, 11 famílias e sete ordens (Figura 3). Os exemplares apresentaram o comprimento padrão entre 12,79 e 96,89 mm (Tabela 1). A ordem mais especiosa foi Perciformes (4 espécies), seguida Blenniformes, Gobiiformes, Tetraodontiformes (2 espécies) e as ordens Anguiliformes, Gobiesociformes e Mugiliformes apresentaram apenas uma espécie. Blennidae e Tetraodontidae foram as famílias mais especiosas, cada uma com duas espécies, enquanto as demais famílias foram representadas por apenas uma. A espécie Lutjanus jocu (Perciformes, Lutjanidae) foi a mais abundante ( $\mathrm{N}=189$ ), seguida por Omobranchus punctatus (Blenniformes, Blennidiae) $(\mathrm{N}=89)$, Bathygobius soporator (Gobiiformes, Gobiidae) $(\mathrm{N}=55)$ e Mugil cf. curema (Mugiliformes, Mugilidae) $(\mathrm{N}=45)$.

As espécies com menor abundância foram Butis koilomatodon (Gobiiformes, Eleotridae) $(\mathrm{N}=10)$, Abudefduf saxatilis (Perciformes, Pomacentridae) ( $\mathrm{N}=7$ ), Gobiesox cf. barbatulus (Gobiesociformes, Gobiesocidae) $(\mathrm{N}=4)$, Genyatremus luteus (Perciformes, Haemulidae) (N $=3$ ), Sphoeroides testudineus (Tetraodontiformes, Tetraodontidae) $(\mathrm{N}=2)$, Gymnothorax funebris (Anguiliformes, Muraenidae) $(\mathrm{N}=2)$, Scartella cristata (Bleniiformes, Bleniidae) $(\mathrm{N}=1)$, Epinephelus itajara (Perciformes, Serranidae) (N = 1) e Colomesus psittacus (Tetraodontiformes, Tetraodontidae) $(\mathrm{N}=1)$.

A baixa quantidade de exemplares capturados no presente estudo, bem como a baixa riqueza de espécies podem estar relacionadas à natureza das poças, pois normalmente as poças da praia do Araçagy possuem profundidade e áreas pequenas, que podem resultar na diminuição de recursos disponíveis (e.g. esconderijo, alimento), no aumento dos estresses fisiológicos (e.g. evaporação da água das poças, aumento da salinidade, aumento da concentração de amônia) e aumentando a seletividade para a presença das espécies mais adaptadas (Nunes et al. 2011). Desta forma, a comparação da ictiofauna da praia do Araçagy seria compatível aos resultados obtidos por Barreiros et al. (2004), Machado et al. (2015) e Pastro et al. (2016), que também encontraram baixa riqueza de espécies em função da sua configuração espacial das poças de maré, divergindo dos padrões encontrados em outros estudos realizados no Brasil.

Por outro lado, nosso estudo destoa dos resultados encontrados em pesquisas pretéritas, que obtiveram maior riqueza de espécies. Possivelmente, as diferenças encontradas na riqueza de espécies em estudos de ictiofauna de poças maré tem sido em função das suas dimensões no volume e área (e.g. volume de água, complexidade estrutural do habitat), pois enquanto em alguns locais do litoral brasileiro os estudos são realizados em pontos com pouco volume de água represada, poças rasas ou mesmo em áreas pequenas (poças de maré: Barreiros et al. 2004, Machado et al. 2015, Pastro et al. 2016), outros estudos foram realizados em locais com volumes consideráveis de água represada, maior profundidade e áreas maiores (piscinas de maré: Rosa et al. 1997, Cunha et al. 2008, Godinho \& Lotufo, 2010, Dantas etal. 2014, Bezerra et al. 2017). Embora essa lógica pareça ser razoavelmente compreensível, tem implicado em certa confusão conceitual e metodológica para os referidos estudos.

Neste sentido, Andrades et al. (2018) conduziram um estudo padronizado com a ictiofauna de poças de maré em toda a extensão do litoral brasileiro, e associaram a riqueza de espécies de peixes poças de maré da praia do Araçagy à baixa complexidade de habitat. Além disso, mencionaram que a variação na salinidade em função dos altos índices pluviométricos da região amazônica possui papel relevante para a ictiofauna da província biogeográfica dos Manguezais Amazônico. A complexidade de habitat das poças também 


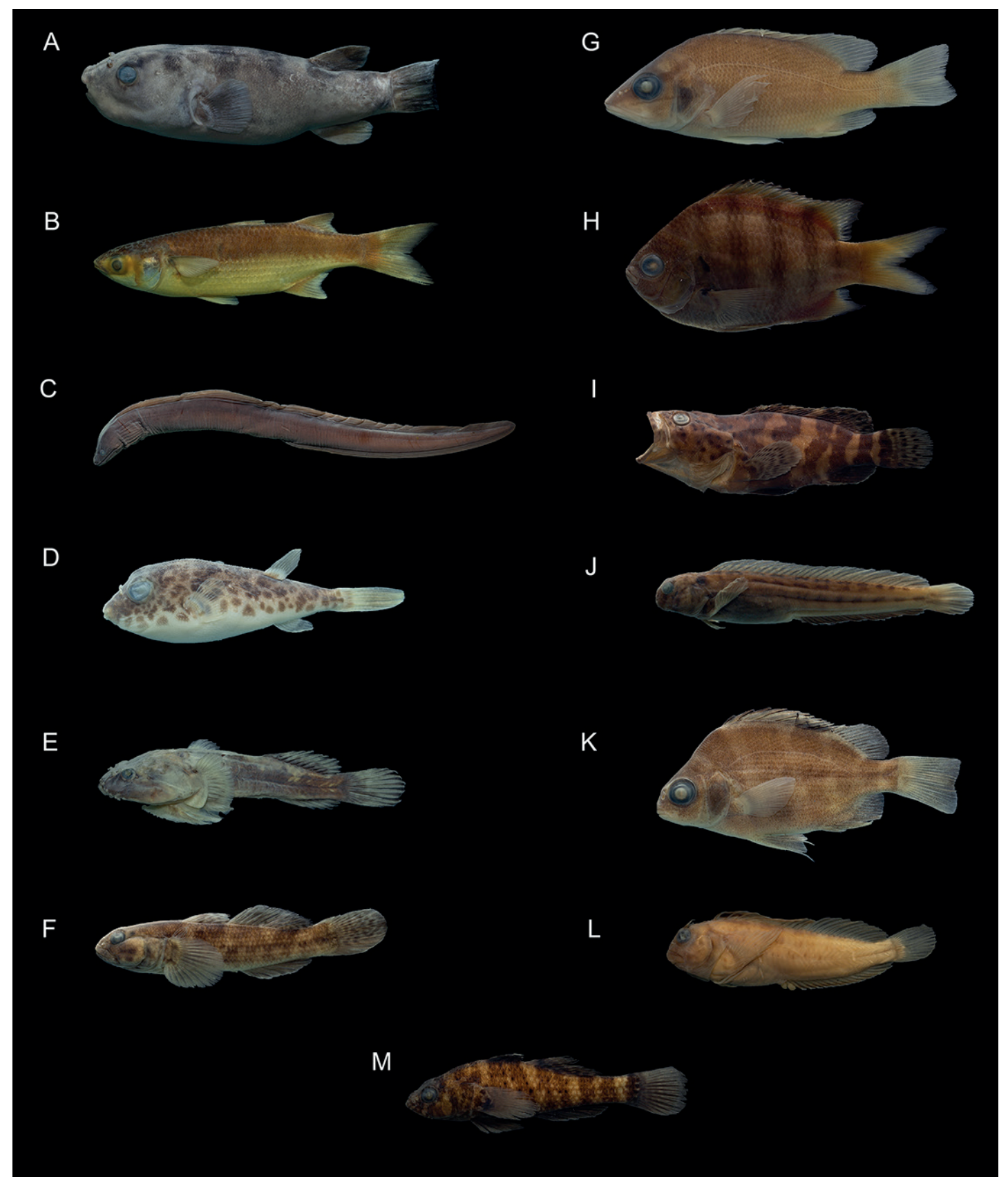

Figura 3. Peixes coletados nas poças de maré na Praia do Araçagy, município de São José de Ribamar, Estado do Maranhão, nordeste do Brasil. A- Colomesus psittacus, 148,3 mm (CICCAA 00729); B- Mugil cf. curema, 122,5 mm (CICCAA 00729); C- Gymnothorax funebris, 251,9 mm (CICCAA 00609); D- Sphoeroides testudineus, 36,3 mm (CICCAA 00608); E- Gobiesoxcf. barbatulus, 43,7 mm (CICCAA 00607); F- Bathygobius soporator, 76,9 mm (CICCAA 00605); G- Lutjanus jocu, 73,7 mm (CICCAA 00612); H- Abudefduf saxatilis, 74,6 mm (CICCAA 00610); I - Epinephelus itajara, 80,5 mm (CICCAA 00878); J - Omobranchus punctatus, 40,9 mm (CICCAA 00740); K - Genyatremus luteus, 54,6 mm (CICCAA 00740); L- Scartella cristata, 31,9 mm (CICCAA 00891) e M- Butis koilomatodon, 41,0 mm (CICCAA 00400).

Figure 3. Fish collected in tide pools at Araçagybeach, municipality of São José de Ribamar, State ofMaranhão, northeastern Brazil. A- Colomesus psittacus, $148.3 \mathrm{~mm}$ (CICCAA 00729); $\mathrm{B}$ - Mugil cf. curema, $122.5 \mathrm{~mm}$ (CICCAA 00729); C- Gymnothorax funebris, $251.9 \mathrm{~mm}$ (CICCAA 00609); D- Sphoeroides testudineus, 36.3 $\mathrm{mm}$ (CICCAA 00608); E- Gobiesox $c f$. barbatulus, $43.7 \mathrm{~mm}$ (CICCAA 00607); F- Bathygobius soporator, $76.9 \mathrm{~mm}$ (CICCAA 00605); $\mathrm{G}$ - Lutjanus jocu, $73.7 \mathrm{~mm}$ (CICCAA 00612); $\mathrm{H}$ - Abudefduf saxatilis, $74.6 \mathrm{~mm}$ (CICCAA 00610); I - Epinephelus itajara, $80.5 \mathrm{~mm}$ (CICCAA 00878); $\mathrm{J}$ - Omobranchus punctatus, $40.9 \mathrm{~mm}$ (CICCAA 00740); K - Genyatremus luteus, $54.6 \mathrm{~mm}$ (CICCAA 00740); L- Scartella cristata, $31.9 \mathrm{~mm}$ (CICCAA 00891) e $M$ - Butis koilomatodon, $41.0 \mathrm{~mm}$ (CICCAA 00400). 
Tabela 1. Lista taxonômica, número de indivíduos (N), comprimento padrão (CP) dos peixes de poças de maré da praia do Araçagy, São José de Ribamar, Maranhão.

Table 1. Taxonomic list, number of individuals ( $N$ ), standard length (CP) of fishes from tidepool at Araçagy beach, São José de Ribamar, Maranhão.

\begin{tabular}{|c|c|c|c|c|c|c|}
\hline $\begin{array}{l}\text { CLASSE/ORDEM/FAMÍLIA/ } \\
\text { ESPÉCIES }\end{array}$ & $\mathbf{N}$ & $\mathbf{C P}(\mathbf{m m})$ & Média & Peso (g) & Média & Vouchers \\
\hline \multicolumn{7}{|l|}{ CLASSE ACTINOPTERY } \\
\hline \multicolumn{7}{|l|}{ ANGUILLIFORMES } \\
\hline \multicolumn{7}{|l|}{ Muraenidae } \\
\hline $\begin{array}{l}\text { Gymnothorax } \\
\text { Ranzani } 1839\end{array}$ & 2 & $254,5-306,81$ & 280,65 & $\begin{array}{l}36,90- \\
48,54\end{array}$ & 42,72 & $\begin{array}{l}\text { CICCAA00609 } \\
\text { CICCAA00728 }\end{array}$ \\
\hline
\end{tabular}

\section{BLENIIFORMES}

Blenniidae

Omobranchus punctatus

(Valenciennes 1836)

89

$17,1-55,13$

22,02

0,02-1,85

1,1833

Scartella

cristata

(Linnaeus 1758)

31,4

GOBIESOCIFORMES

\section{Gobiesocidae}

Gobiesox cf. barbatulus

4

$42,74-53,39$

46,82

$0,97-2,51$

1,53

CICCAA00607, CICCAA00739

\section{GOBIIFORMES}

\section{Eleotridae} Butis koilomatodon
(Bleeker 1849)

10

$12,50-76,52$

50,38

0,04-4,73

2,094

CICCAA00400, CICAA00732,

CICAA00735

\section{Gobiidae}

Bathygobius soporator (Valenciennes 1837)

55

$34,4-96,89$

167,09

0,89-10,5

4,19

CICCAA00605, CICCAA00606, CICAA00737, CICCAA00793

\section{MUGILIFORMES}

\section{Mugilidae}

Mugil cf. curema

45

$17,68-125,23$

106,05

0,07-3,88

2,54

CICCAA00603, CICCAA00604, CICCAA00729, CICCAA00736

\section{PERCIFORMES}

\section{Haemulidae}

Genyatremus (Bloch 1790)

luteus $3 \quad 22,72-69,80$ 41,27

$0,03-5,52$ 2,01

CICCAA00740

\section{Lutjanidae}

Lutjanus jocu (Bloch \& Schneider 1801)
189

$19,03-99,80$

45,015

4,26
$0,09-9,81$
ICCAA00611, CICCAA00612, CICCAA00730, CICAA00733 
Tabela 1....continuação

Table 1....continued

\begin{tabular}{|c|c|c|c|c|c|c|}
\hline $\begin{array}{l}\text { CLASSE/ORDEM/FAMÍLIA/ } \\
\text { ESPÉCIES }\end{array}$ & $\mathbf{N}$ & $\mathbf{C P}(\mathbf{m m})$ & Média & Peso (g) & Média & Vouchers \\
\hline \multicolumn{7}{|l|}{ Pomacentridae } \\
\hline $\begin{array}{l}\text { Abudefduf saxatilis } \\
\text { (Linnaeus 1758) }\end{array}$ & 7 & $35,21-99,85$ & 60,19 & $\begin{array}{l}0,73- \\
24,46\end{array}$ & 5,81 & $\begin{array}{l}\text { CICCAA00610, } \\
\text { CICCAA00615, } \\
\text { CICAAA00738 }\end{array}$ \\
\hline \multicolumn{7}{|l|}{ Serranidae } \\
\hline $\begin{array}{l}\text { Epinephelus itajara } \\
\text { (Lichtenstein 1822) }\end{array}$ & 1 & 78,39 & - & - & - & CICCAA00878 \\
\hline \multicolumn{7}{|l|}{ TETRAODONTIFORMES } \\
\hline \multicolumn{7}{|l|}{ Tetraodontidae } \\
\hline $\begin{array}{l}\text { Sphoeroides testudineus } \\
\text { (Linnaeus 1758) }\end{array}$ & 2 & $25,89-44,12$ & 35,00 & $0,36-1,96$ & 1,16 & CICCAA00608 \\
\hline $\begin{array}{l}\text { Colomesus psittacus } \\
\text { (Bloch \& Schneider 1801) }\end{array}$ & 1 & 180,86 & - & - & - & CICCAA00727 \\
\hline
\end{tabular}

parece ter influência direta da dinâmica de praia, pois suas características morfológicas são constantemente modificadas pela ação de ventos, ondas, sedimentos e a maré (Tarouco \& Santos 1997), remodelando constantemente suas características estruturais e físico-química. Além disso, parece que a ausência de ambientes recifais adjacentes às poças de maré da praia do Araçagy também pode resultar na baixa riqueza de espécies, pois a maioria dos estudos sobre a ictiofauna de poças de maré realizados no Brasil possuem influência de ambientes recifais (Rosa et al. 1997, Cunha et al. 2008, Chaves et al. 2010, Godinho et al. 2010, Dantas et al. 2014, Bezerra et al. 2017).

De maneira geral, as famílias Blennidae e Gobiidae são relatadas como tipicamente associadas às poças de maré em todo o mundo (Prochazka etal.1999), e o mesmo padrão também foi observado na assembleia de peixes de poças de maré na praia do Araçagy, onde as espécies O. punctatus e B. soporator foram abundantes. As espécies $L$. jocu e $M$. cf. curema, também foram abundantes no presente estudo, utilizando as poças como área de berçário, e em seguida buscando águas costeiras mais profundas para terminar o ciclo de vida (Machado et al. 2015, Fricke et al. 2021).

Nossos resultados também mostraram que as poças de maré da praia do Araçagy apresentam panoramas muito importantes quanto à conservação das espécies que compõem a assembleia de peixes. Um dos exemplos disso foi a presença de Epinephelus itajara, espécie categorizada como "Vulnerável" na lista vermelha nacional e "Criticamente em Perigo" na lista vermelha internacional (Duailibe et al. 2021), que, embora tenha apenas um registro, sugere que as poças têm sido usadas pelos indivíduos juvenis antes de migrarem para outros ambientes. Também foi observada a presença de espécies não nativas. Omobranchus punctatus foi uma das espécies mais abundantes das poças de maré da Praia do Araçagy e possuiu uma ampla distribuição no ambiente, pois seus indivíduos foram encontradas em vários tipos de poças e em diferentes estratos do médio litoral (Lasso et al. 2011). Por outro lado, a outra espécie de peixe não nativa, B. koilomatodon, foi rara nas praias da Ilha do Maranhão e geralmente apresentaram baixos números de indivíduos (Guimarães et al. 2017, Mendes et al. 2017). Essas informações podem nos sugerir que o uso da estratégia de colonização por B. koilomatodon foi menos eficiente do que a utilizada por O. punctatus, ou processo de introdução ainda incipiente. Contudo, ambos os pontos de vista poderão ser objetos de futuros estudos ecológicos com estas duas espécies nativas do oceano Pacífico.

A ictiofauna de poças de marés da praia do Araçagy apresentou baixa riqueza de espécies. Contudo, parece ser influenciada pelo complexo estuarino do Golfão Maranhense, embora também possa ser possível ter influência dos 
recifes Amazônicos. Além disso, consideramos que é importante direcionar esforços para a compressão das interações ecológicas e para a conservação destes ambientes, que possuem papel fundamental no ciclo de vida de espécies comerciais (e.g. M. cf. curema, G. luteus) e ameaçadas (e.g. E. itajara).

\section{AGRADECIMENTOS}

Nós agradecemos à FAPEMA (Fundação de Amparo à Pesquisa e ao Desenvolvimento Científico e Tecnológico do Maranhão) e à UFMA (Universidade Federal do Maranhão) por proporcionarem a infraestrutura para a realização desse trabalho. Agradecemos também ao Axel Katz pelo auxílio na edição das fotografias e ao Lucas Vieira pela assistência no laboratório.

\section{REFERÊNCIAS}

Andrades, R., Machado, F. S., Reis-Filho, J. A., Macieira, R. M., \& Giarrizzo, T. 2018. Intertidal Biogeographic Subprovinces: Local and Regional Factors Shaping Fish Assemblages. Frontiers in Marine Science, 5. DOI:10.3389/ fmars.2018.00412.

Barreiros, J. P., Bertoncini, Á., Machado, L., Hostim-Silva, M., \& Santos, R. S. 2004. Diversity and seasonal changes in the ichthyofauna of rocky tidal pools from Praia Vermelha and São Roque, Santa Catarina, Brazil. Brazilian Archives of Biology Technology, 47(2), 291-299. DOI: 10.1590/S1516-89132004000200017.

Bezerra, L. A. V., Padial, A. A., Mariano, F. B., Garcez, D. S., \& Sánchez-Botero, J. I. 2017. Fish diversity in tidepools: assembling effects of environmental heterogeneity. Environmental Biology of Fishes, 100(5), 551-563. DOI: 10.1007/ s10641-017-0584-3.

Carpenter, K. E. 2002a. The living marine resources of the Western Central Atlantic. Vol. 2: Bony fishes part 1 (Acipenseridae to Grammatidae) FAO Species Identification Guide for Fishery Purposes and American Society of Ichthyologists and Herpetologists Special Publication. No 5. Rome, FAO. p. 1373.

Carpenter, K.E. 2002b. The living marine resources of the Western Central Atlantic. Vol. 3: Bony fishes part 2 (Opistognathidae to Molidae), sea turtles and marine mammals. FAO Species Identification Guide for Fishery Purposes and American Society of Ichthyologists and Herpetologists Special Publication. No 5. Rome, FAO. p. 2127.

Chaves, L. D. C., Nunes, J. D. A. C., \& Sampaio, C. L. 2010. Shallow reef fish communities of South Bahia coast, Brazil. Brazilian Journal of Oceanography 58(special issue IICBBM), 3346.DOI: 10.1590/S1679-87592010000800006.

Cunha, F.E.D.A., Monteiro-Neto, C., \&Nottingham, M. C. 2007. Temporal and spatial variations in tidepool fish assemblages of the northeast coast of Brazil. Biota Neotropica, 7(1), 95-102. DOI: 10.1590/S1676-06032007000100016.

Cunha, E. A., Carvalho, R. A., Monteiro-Neto, C., Moraes, L. E. S., \& Araújo, M. E. 2008. Comparative analysis of tidepool fish species composition on tropical coastal rocky reefs at State of Ceará, Brazil. Iheringia, Série Zoológica, 98(3), 379-390. DOI: 10.1590/S007347212008000300013 .

Dantas, R. S., Dantas, P. S. B., \& da Costa Ramos R. T. 2014. Ictiofauna das piscinas de maré da Praia do Paiva: Um registro anterior à exploração imobiliária. Tropical Oceanography 42(2), 253260. DOI: https://10.5914/to.2014.01186.

Duailibe, I. C. F. S., Coelho, K. K. F., Filgueira, C. H. M. S., Palmeira-Nunes, A. R. O., Saraiva, A. C. S., \& Nunes, J. L. S. 2021. Uso de mídias digitais aplicado à estudos de conservação do mero Epinephelus itajara (Lichtenstein, 1822) no Litoral Amazônico Brasileiro. Boletim do Laboratório de Hidrobiologia, 31, 1-8.

Freitas, J. E. P., \& Lotufo, T. M. D. C. 2015. Reef fish assemblage and zoogeographic affinities of a scarcely known region of the western equatorial Atlantic. Journal of The Marine Biological Association of The United Kingdom, 95(3), 623-633. DOI: 10.1017/ S0025315414001404.

Fricke, R., Eschmeyer, W. N., \& Fong, J. D. 2021. SPECIES BY FAMILY/SUBFAMILY. (http:// researcharchive.calacademy.org/research/ ichthyology/catalog/SpeciesByFamily.asp). Acessado eletronicamente em 22 de abril de 2021.

Godinho, W. O., \& Lotufo, T. M. C. 2010. Microhabitat influences on the fish fauna of tidal pools in north-east Brazil. 
Journal of Fish Biology 76(3), 487-501. DOI: 10.1111/j.1095.2009.02301.x.

Guimarães, E. C., Brito, P. S., \& Ottoni, F. P. 2017. First record of Butis koilomatodon (Bleeker, 1849) (Gobiiformes: Eleotridae) for the Maranhão state, northeastern Brazil: a case of bioinvasion. Cybium, 41(3), 299-300.

Lasso-Alcalá, O., Nunes, J. L., Lasso, C., Posada, J., Robertson, R., Piorski, N. M., \& Gondolo, G. 2011. Invasion of the Indo- Pacific blenny Omobranchus punctatus (Perciformes: Blenniidae) on the Atlantic Coast of Central and South America. Neotropical Ichthyology, 9(3), 571-578. DOI: 10.1590/S167962252011000300010 .

Macieira, R. M., \& Joyeux, J. C. 2011. Distribution patterns of tidepool fishes on a tropical flat reef. Fishery Bulletin, 109(3), 305-315.

Machado, F. S., Macieira, R. M., Zuluaga Gómez, M. A., Costa, A. F., Mesquita, E., \& Giarrizzo, T. 2015. Checklist of tidepool fishes from Jericoacoara National Park, south western Atlantic, with additional ecological information. Biota Neotropica, 15(1), 1-9. DOI: 10.1590/1676-06032015011114.

Mendes, M. B. P., Martins, A. P. B., Coelho, G.K.F, Piorski, N. M., \& Nunes, J. L. S. 2017. Non-native mud sleeper Butis koilomatodon (Bleecker, 1849) (Perciformes: Eleotridae) in Eastern Amazon Coastal region: an additional occurrence for the Brazilian coast and urgency for ecological assessment. Bioinvasion Records, 6, 111-117.

Nunes, J. L. S., Pascoal, N. G. A., \& Piorski, N. M. 2011. Peixes intertidais do Maranhão. In: Nunes, J., Pascoal, N. \& Piorski, N. (Eds.). Peixes marinhos e estuarinos do Maranhão. pp. 105-124. Editora Café e Lápis.

Pastro, G., Dias, G. M., \& Gibran, F. Z. 2016. Structure and composition of fish assemblages from São Sebastião Channel tide pools, southwestern Atlantic. Biota Neotropica, 16(3), 1-9. DOI: 10.1590/1676-0611-bn-2015-0128.

Prochazka, K., Chotkowski, M., \& Buth, D. 1999. Biogeography of rocky intertidal fishes. In: Horn, M. H., Martin, K. L. M. \& Chotkowski, M. A., (Eds.). Intertidal fishes: life in two worlds. p. 332-355. Elsevier.

Rosa, R. S., Rosa, I. L., \& Rocha, L. A. 1997. Diversidade da ictiofauna de poças de maré da praia do Cabo Branco, João Pessoa, Paraíba, Brasil. Revista Brasileira de Zoologia, 14(1), 201-212.

Tarouco, J. E., Santos, J. H. S dos. 1997. Morfodinâmica da Praia do Araçagi. Paço do Lumiar-MA. $1^{\circ}$ Fórum Latino Americano de Geografi a Física Aplicada. Anais, p. 1-7. Curitiba-PR.
Submitted: 25 January 2021 Accepted: 9 July 2021

Published on line: 27 July 2021 Associate Editor: José Luis Novaes 